\title{
Comparative analysis for evaluating the traceability of interventional devices using blood vessel phantom models made of PVA-H or silicone
}

\author{
Chang-Ho Yu ${ }^{\mathrm{a}}$, Tae-Kyu Kwon ${ }^{\mathrm{a}, *}$, Chan Hee Park ${ }^{\mathrm{b}, *}$, Makoto Ohta ${ }^{\mathrm{c}}$ and Sung Hoon Kim${ }^{\mathrm{d}, *}$ \\ ${ }^{a}$ Division of Biomedical Engineering, Chonbuk National University, Jeonju-si, Jeonbuk, Korea \\ ${ }^{\mathrm{b}}$ Division of Mechanical Design Engineering, Chonbuk National University, Jeonju-si, Jeonbuk, Korea \\ ${ }^{\mathrm{c}}$ Institute of Fluid Science, Tohoku University, Sendai, Miyagi, Japan \\ ${ }^{\mathrm{d}}$ Department of Electronics Convergence Engineering, Wonkwang University, Iksan-si, Jeonbuk, Korea
}

\begin{abstract}
.
OBJECTIVE: In this paper, we investigated the parameters with effective traceability to assess the mechanical properties of interventional devices.

METHODS: In our evaluation system, a box-shaped poly (vinyl alcohol) hydrogel (PVA-H) and silicone were prepared with realistic geometry, and the measurement and evaluation of traceability were carried out on devices using load hand force. The phantom models had a total of five curve pathways to reach the aneurysm sac.

RESULTS: Traceability depends on the performance of the interventional devices in order to pass through the curved part of the model simulation track. The traceability of the guide wire was found to be much better than that of the balloon and stent loading catheter, as it reached the aneurysm sac in both phantom models.

CONCLUSIONS: Observation using the video record is another advantage of our system, because the high transparency of the materials with silicone and PVA-H can allow visualization of the inside of an artery.
\end{abstract}

Keywords: Blood vessel phantom model, PVA-H, traceability, catheter, guide wire

\section{Introduction}

Catheters are used for drug administration, to observe, diagnose, or treat infarctions, aneurysms, and stenosis, or for implantation in endovascular surgery (Embolization). Endovascular treatment using a catheter was recently revealed as a minimally invasive treatment model [1-3], since which time the

\footnotetext{
${ }^{*}$ Corresponding authors: Tae Kyu Kwon, Division of Biomedical Engineering, Chonbuk National University, 567 Baekjedaero, Deokjin-gu, Jeonju-si, Jeonbuk 561-756, Korea. Tel.: +82 63270 4066; Fax: +82 63270 2247; E-mail: kwon10@ jbnu.ac.kr. Chan Hee Park, Division of Mechanical Design Engineering, Chonbuk National University, 567 Baekje-daero, Deokjin-gu, Jeonju-si, Jeonbuk 561-756, Korea. Tel.: +82 63270 2365; Fax: +82 63270 0311; E-mail: biochan@jbnu.ac.kr. Sung Hoon Kim, Department of Electronics Convergence Engineering, Wonkwang University, 460 Iksan-daero, Iksan-si, Jeonbuk 570-749, Korea. Tel.: +82-63-850-6739; Fax: +82 63850 6739; E-mail: kshoon@wku.ac.kr.
} 
number of cases of endovascular treatment implementing catheters has been increasing. Surgeons manipulate the catheter using an angiography monitor in order to position it at the disease site. The supply roller was developed to provide surgical instruments to edge the catheter away from a fluoroscope, and the catheter was further developed as a tool for tele operation using LSM [4-6]. Similarly, the use of ACIS can reduce radiation exposure to both surgeons and patients. The latest model of MMCS has a small size, only $0.8 \mathrm{~mm}$ in diameter and $8 \mathrm{~mm}$ in length. The MMCS is used for needle guidance [7], bronchoscopic manipulation [8], and laparoscopic surgery manipulation [9]. The MMCS was compared with other corrective methods through use of a mathematical method by Kindratenko and Bennett [10]. Another method using an optical detector was presented for co-calibration between sensors [8 11112].

However, only a few studies have investigated catheter performance, most of which were small-scale clinical studies [13-17]. In addition, in-vitro studies on micro catheters are even rarer, owing to the difficultly evaluating the performance and characteristics of micro catheters [18].

A box-shaped in-vivo model can provide stable conditions for interventional training, and is also convenient for the technical assessment of medical devices [19]. This is attributable to its stable geometric structure. As PVA-H (Poly vinyl alcohol hydrogel) can be formed according to the desired design, a box-shaped PVA-H model resembling the vascular lumen was developed. Assessment of the mechanical characteristics of interventional devices such as guide wires, catheters, balloons, and stents are required to provide experience to surgeons, and to provide an accurate assessment system for new devices to medical device developers.

To characterize the mechanical properties of interventional devices, traceability was defined as the device's ability to easily move and trace the target via a curved vascular route. To assess the mechanical characteristics of interventional devices, a box-shaped PVA-H with an actual geometric structure was prepared, and its traceability was measured and assessed using the in-vitro tracing system.

\section{Experimental methods}

\subsection{Poly (vinyl alcohol) hydrogel phantom model}

To construct a realistic mold, it is prepared with combination of clinical imaging method, rapid typing technique and coating of polymer, models of the cerebral vasculature shown in Fig. 3(a) were created from patients undergoing clinically indicated conventional angiography with rotational data acquisition. Figure 3(b) shows a model made of gypsum. A material of biomodel is deposited on the mold by various technique (pouring, painting, dip-coating, etc.), and at last the mold is removed by lost-wax technique (heating, chemically disolving, etc.).

A phantom model made of poly (vinyl alcohol) hydrogel (PVA-H) was developed to overcome the previous weaknesses. PVA-H is mainly composed of poly (vinyl alcohol) (PVA) and water. It may also include other organic solvent, such as dimethyl sulfoxide (DMSO). This hydrogel is made by utilizing the characteristics of PVA to be easily dissolved in water or other hydrophilic solvents. Because PVA has many hydrophilic groups (hydroxyl groups) in its molecular components (Fig. 1), the polymer chain of PVA can bond to water or other hydrophilic molecules. The characteristics of PVA were researched energetically in the 1960s, and many synthesis methods and applications were developed. Gelation of PVA is one of those applications, and the PVA hydrogel is a beneficial gel which can also be applied in the medical field [20]. Most PVA hydrogels are obtained by dissolving PVA powder in water at high temperatures, after which freeze-thaw cycles are carried out to provide high strength to the gel [21]. However, PVA hydrogels obtained by freeze-thaw cycles are not usually transparent. In the late 1980s, 


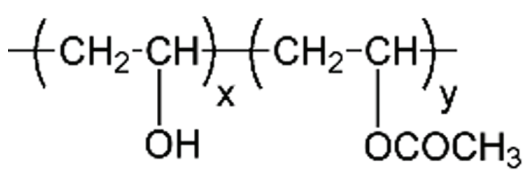

$\mathrm{DP}=\mathrm{x}+\mathrm{y}$

$\mathrm{SV}=\mathrm{x} /(\mathrm{x}+\mathrm{y})^{*} 100$

(a)

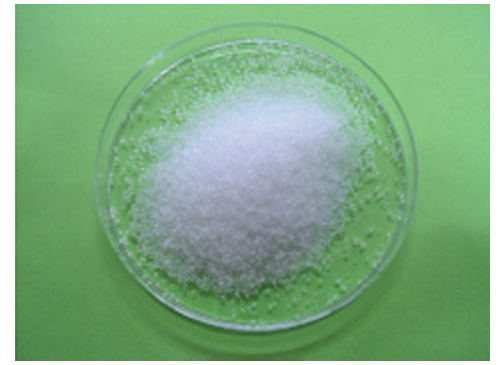

(b)

Fig. 1. (a) Chemical structure of PVA, and (b) PVA powder. DP is the degree of polymerization, and SV is the saponification value. PVA has a hydrophilic group (hydroxyl group) in the unit with the subscript $x$, and a hydrophobic group (carboxyl group) in that with the subscript $y$ in its structure. For general information, the number of $x$ is larger than $y$, and SV becomes higher than $80 \%$.

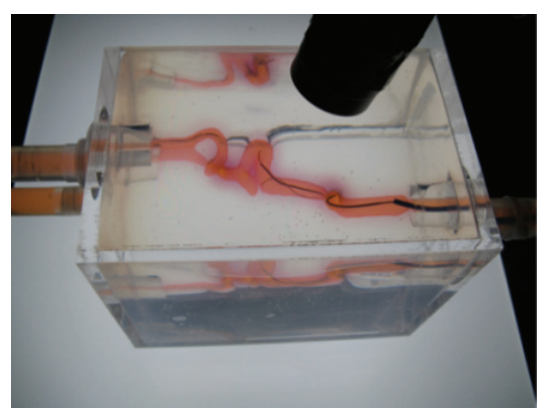

(a)

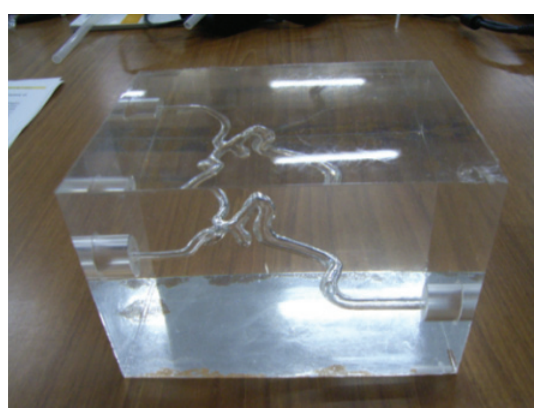

(b)

Fig. 2. (a) The box-shaped PVA-H phantom model with the actual geometry. (b) The box-shaped silicone phantom model with the actual geometry, which replicated the PVA-H boxed model.

Hyon et al. reported a strong, transparent PVA gel could be obtained by using DMSO aqueous solution as the solvent [22]. According to their report, the transparency and tensile strength of the resulting gel is variable due to the concentration of DMSO in the aqueous solution, and PVA hydrogels composed of $80 \mathrm{wt} \%$ of DMSO aq. are the strongest and most transparent.

The components (DP, SV) of the PVA polymer chain can be changed during chemical processing. Ohkura et al. reported that the molecular component of PVA affects the sol-gel transition curve [23]. This result indicates that the molecular component of PVA also affects the physical and chemical characteristics of the PVA hydrogel. In addition, the physical characteristics of the PVA gel are also affected by the facticity of polymer structure [24]. The PVA-H model is shown in Fig. 2(a).

\subsection{Experimental system for traceability testing}

A three-dimensional pathway that simulated the intracranial carotid artery was constructed from PVA$\mathrm{H}$ and silicone. In this study, traceability was considered as the ability of the interventional devices to track or move easily through a curved vascular pathway. To characterize the traceability, the proximal push force by hand required to advance the catheters, balloon and guide wires through the in vitro phantom model made of PVA-H or silicone was measured. Measurements were performed using seven different interventional devices ( 1 balloon, 4 stent-loading catheters and 2 guide wires) to evaluate the 


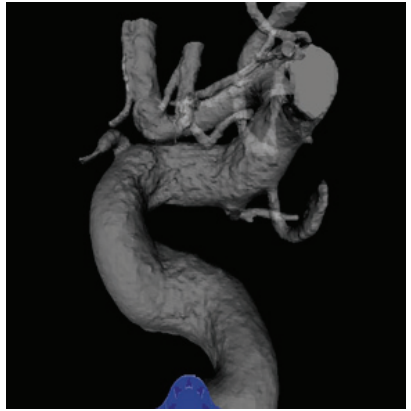

(a)

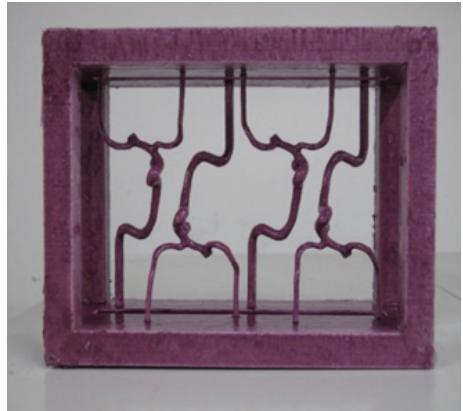

(b)

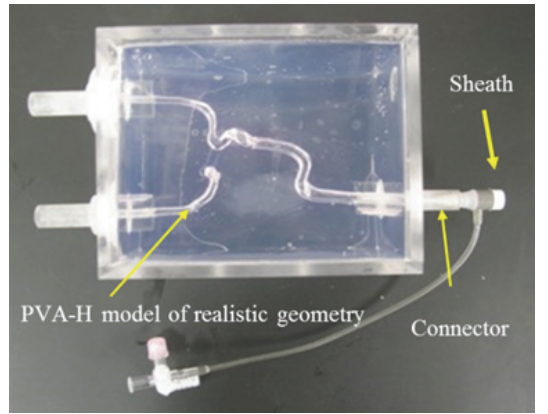

(c)

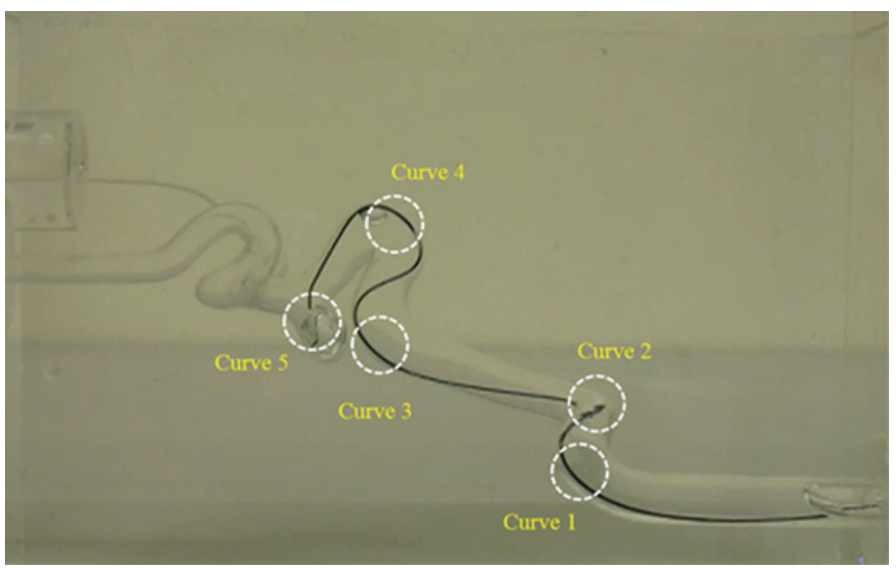

(d)

Fig. 3. (a) Three-dimensional model of a patient is prepared with a combination of clinical imaging, rapid typing technique, and coating of polymer. Models of the cerebral vasculature were created from patients undergoing clinically indicated conventional angiography with rotational data acquisition. (b) The 3-D geometry was transferred to a model made of gypsum. (c) The circulatory tracking system shown consists of a PVA-H model, an acrylic mold of that model, a sheath and a connecter [25]. (d) Box-shaped in vitro phantom model mimicking the geometry of a human intracranial artery. This phantom model had a total of five curve pathways to reach the aneurysm sac. A guide wire was inserted from the right (common carotid artery) inlet part.

traceability. All measurements were performed for a single device of each interventional device brand. The stent-loading catheters (TaeWoongMedical Co., Ltd., Korea) had stents of different porosity on the tips $(64 \%, 71 \%, 74 \%$ and $80 \%)$. The guide wires tested included a single 0.016 inch Glidewire GT (Terumo Co., Tokyo, Japan) and a single 0.016 inch Gateway (Boston scientific, Natick, MA, USA). Traceability is essentially a measure of the resistance against the devices moving through a curved vessel pathway. The lower the push forces felt, the better the traceability. Validation measurements have established that the number of curves is responsible for the difficulty level of the relating model path [26]. The tracking system tested consisted of a PVA-H phantom model or silicon phantom model, an acrylic mold of the model, a sheath, and a connecter. These in vitro phantom models had a total of five curvatures, as shown in Fig. 3(d). All devices were inserted into the tracking systems by hand force, and the motion was recorded with a digital camera.

\section{Results}

The in vitro tracking system that was developed in the present study, described in detail in Fig. 3(c), 


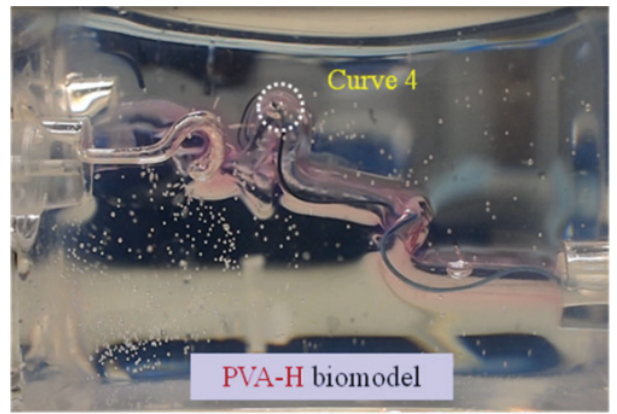

(a)

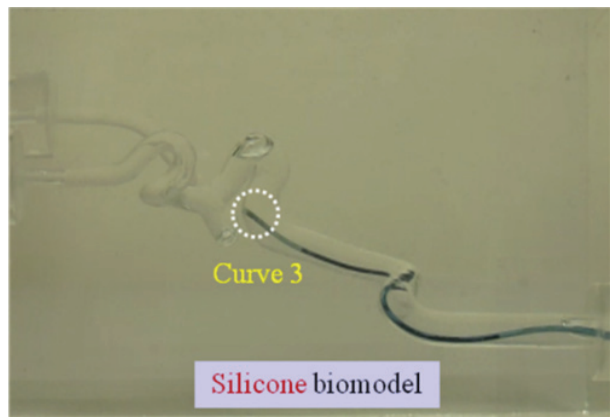

(c)

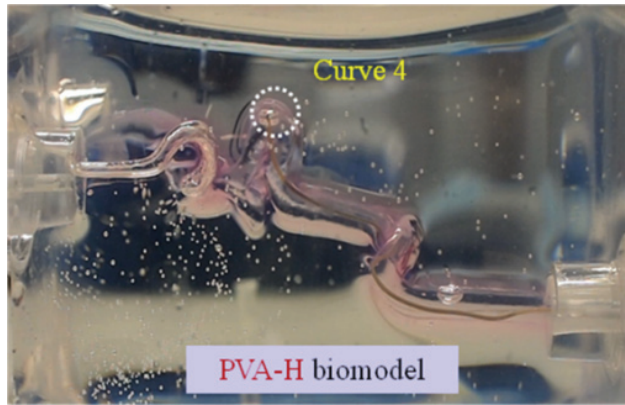

(b)

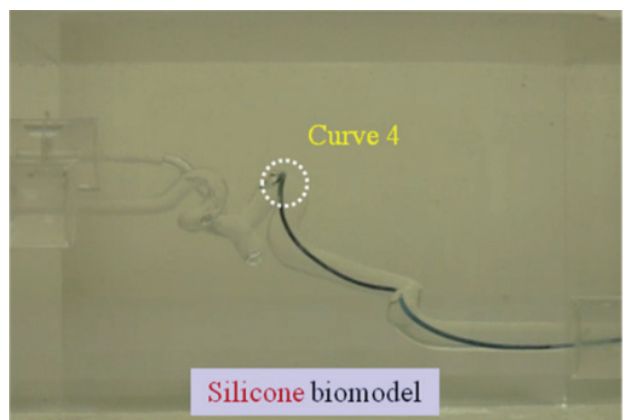

(d)

Fig. 4. (a) Motion capture for evaluating traceability of a stent loading catheter in the PVA-H phantom model (Stent porosity: $80 \%$ ). (b) Motion capture for evaluating traceability of a stent loading catheter in the silicone phantom model (Stent porosity: 80\%). (c) Motion capture for evaluating traceability of a stent loading catheter in the PVA-H phantom model (Stent porosity: $64 \%$ ). (d) Motion capture for evaluating traceability of a stent loading catheter in the silicone phantom model (Stent porosity: $64 \%)$.

was evaluated for traceability. Validation measurements have established that the number of curves is responsible for the difficulty level of the related model path. Each device was advanced through this tortuous pathway with the endpoint being measured as the maximal distance traversed by the devices. As shown in Figs 4 and 5, our phantom model made of PVA-H or silicone was very clear to provide an understanding of the catheter, balloon and guide wire movement. They could be inserted into the artery smoothly.

The traceability of the guide wires was much better than that of the balloon and stent loading catheter, as only they reached the aneurysm sac. Markedly, the Gateway guide wire (Boston scientific, Natick, MA, USA) was the most deliverable, reaching curve 5 in both the phantom model in Figs 5(a) and (b). On the other hand, the other guide wire tested, Glidewire GT (Terumo Co., Tokyo, Japan), produced significantly different results. As shown in Figs 5(c) and (d), it could only reach curve 5 in the PVA-H phantom model, but failed in the silicone phantom model.

These results suggest that the stents with higher porosity had higher stiffness. This phenomenon can be explained by the higher friction coefficient of silicone and its higher stiffness, which made it difficult to control compared to the PVA-H phantom model. For a given vessel wall architecture, the differences in traceability must arise from differences in the endovascular devices; such differences most likely originate from differences in the surfaces of the devices, such as surface roughness, composition, or actual contact area among others, giving rise to frictional resistance. The bending stiffness of the stent system will also contribute to the traceability. 


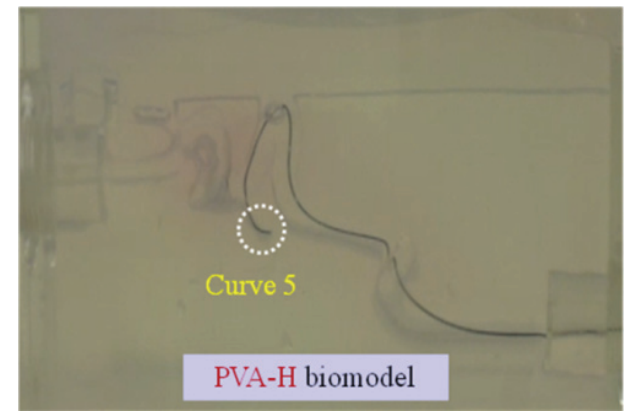

(a)

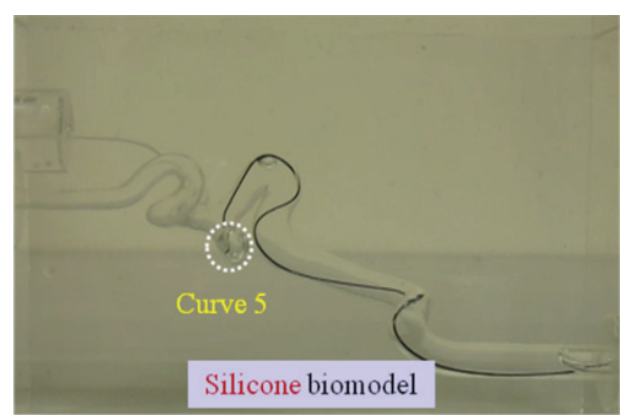

(c)

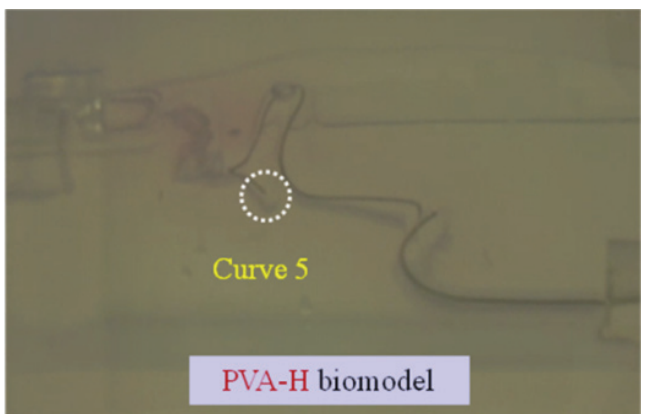

(b)

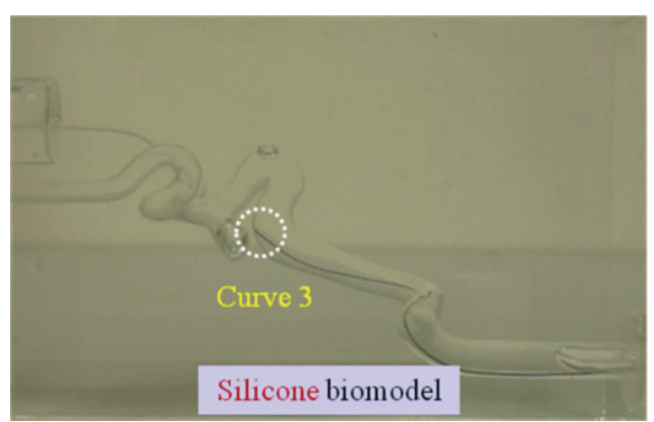

(d)

Fig. 5. (a) Motion capture for evaluating traceability of a guide wire (Bostion scientific, Gateway) in the PVA-H phantom model. (b) Motion capture for evaluating traceability of a guide wire (Bostion scientific, Gateway) in the silicone phantom model. (c) Motion capture for evaluating traceability of a guide wire (Terumo, Glidewire GT) in the PVA-H phantom model. (d) Motion capture for evaluating traceability of a guide wire (Terumo, Glidewire GT) in the silicone phantom model.

A stiffer guide wire and micro catheter system may transmit force more efficiently because it will act more like the example of a rigid rod; however, a stiffer system will also not conform to a curved segment of a vessel, and hence can potentially result in greater contact forces, which will reduce the force transmission and hence the traceability.

The most striking difference was observed in terms of the device's abilities to reach the predetermined region in both the PVA-H and silicone phantom models by using in vitro tracking system developed herein. The best traceability was observed for the Gateway guide wire (Boston scientific, Natick, MA, USA) in both the PVA and silicone phantom models. As shown in Figs 5(a) and (b), this guide wire was able to consistently reach the lesion in both phantom models while the other devices failed. This is likely because the guide wire had a more gradual transition in stiffness, from a floppy tip to a stiffer body. Table 1 shows the comparison of our system with previous research.

\section{Discussion}

In this study, a leading effort was made to define and measure in-vitro objective mechanical variables in order to compare the mechanical characteristics of six interventional devices which are currently commercially available. It is no doubt that actual clinical conditions are more complex than in-vitro conditions. However, in-vitro simulation is considered very useful for understanding the physical 


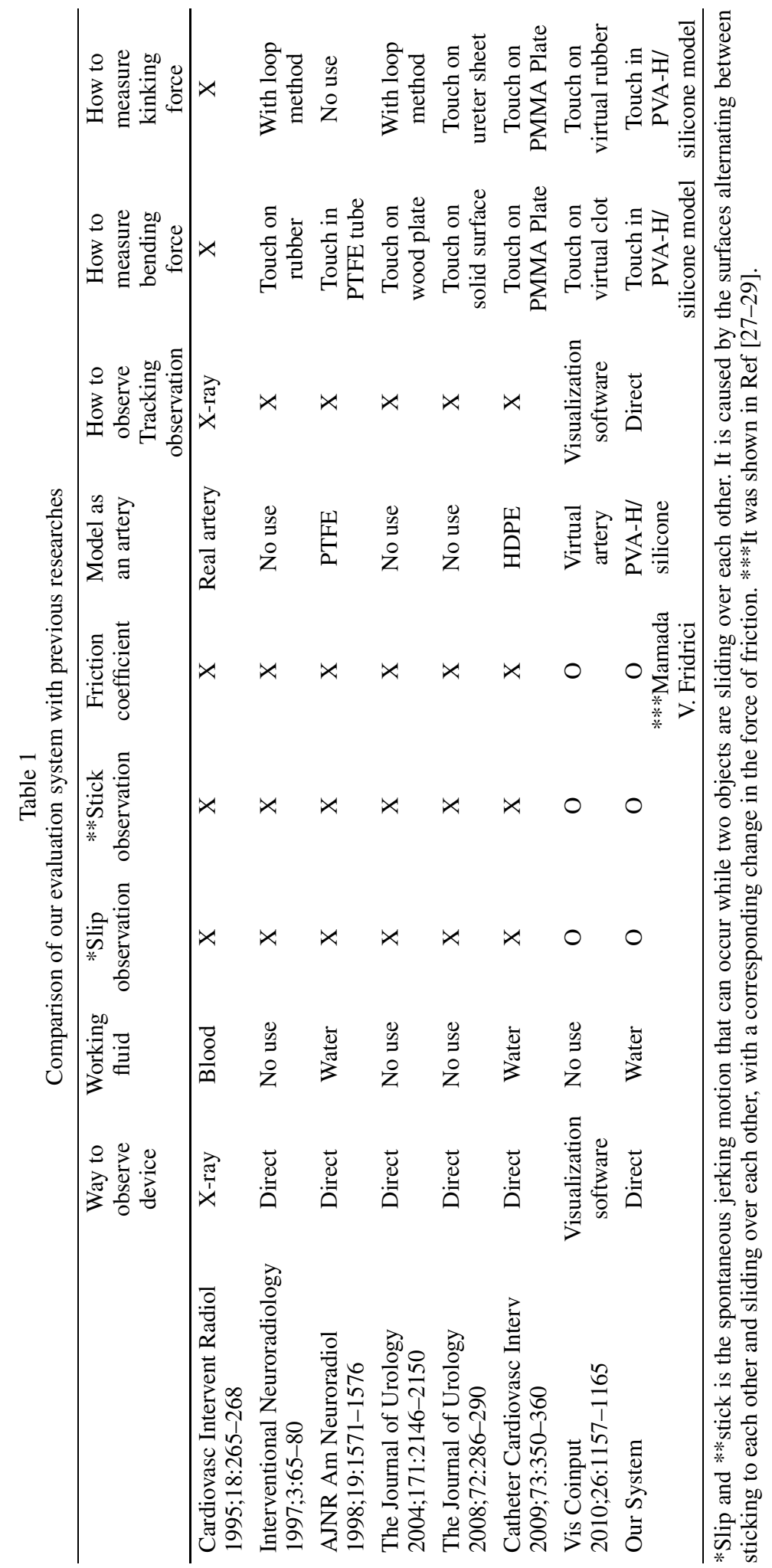


characteristics of interventional devices, as well as for providing the best guidelines to clinicians. Although good outcomes obtained from in-vitro studies do not guarantee safety and ease of the approaches in-vivo with subjects, poor outcomes obtained from in-vitro studies have shown a high possibility to cause difficulty in complex lesions or risk of complications.

Various techniques have been used in clinical practices to promote the technical improvement of the micro catheter/guide wire combination. One of the most commonly used techniques is to use the loose part of the catheter that reaches the winding vascular distribution by gently re-inserting the guide wire after it was retreated sufficiently. Factors that affect the performance of devices inserted into blood vessels include smoothness, rigidity, and durability. Device manufactures have employed various catheter materials, hydrophilic coatings, and mechanical designs to optimize the safety and traceability of catheters. It is difficult to design an in-vitro model that stimulates cranial carotid artery circulation without fatigue or change during use. The shape of a PVA-H model was designed to impose reasonable frictional resistance, and to stimulate the curvature of cranial carotid artery circulation during catheter insertion. A similar model might be made using a dead body or animal artery. However, such a model is difficult to standardize during the long-term period of repeated test procedure. Furthermore, test errors may occur due to the use of such models. The PVA-H model is considered to provide a reasonable material that can build a route that will not confuse the measurement of catheter performance despite the poor expanding ability of the in-vitro arterial segment.

Three delivery variables (pushing ability, traceability, and cross-over) based on resistance can be quantified according to the portion of the delivery route. This definition also includes the measurement of force that may be felt or applied by clinicians. Thus, these variables will potentially be able to show clinicians how a specific stent is inserted to the end part of the proximal portion. As artery models become more improved and more precisely upgraded, their definition will progress to reflect the new understanding of stent insertion

Traceability depends on the performance of the interventional devices in order to pass through the curved part of the model simulation track. In addition, the weak pushing force of the proximal portion, known as the load, indicates that the traceability of proximal segment in the insertion (delivery) route is good. The pushing force of the proximal portion constantly increases during the procedure performed via the model. In this study, the optimized traceability was measured using the guidewires of two in-vivo models (Boston scientific, Gateway). The catheter was pushed ahead, reaching the saccular aneurysm of curve 5 (Figs 5(a) and (b)). The performance of the catheter and guide wire, that is, the curving and twisting ability in the winding blood vessel, is likely to depend on "endurable" connectivity rather than on the rigid nodule. The curving ability of the end part of the catheter and guide wire depends on their geometric structure and materials. Traceability and curving ability are likely to be associated with each other. The rigidities of the interventional devices were not precisely characterized in this study. Nonetheless, the aforementioned test method enables the simple, easy, and accurate comparative measurement of traceability which is imperative for clinical implementation.

\section{Conclusion}

The new system developed herein was evaluated in terms of mechanical properties of the interventional devices, and their effects on traceability were determined.

In the current setting, the proposed system provided information about the ability of interventional devices to pass and push through the tortuous pathway made of PVA-H and silicone. 
Other important features of the proposed methodology are its modularity and extensibility. While the current test situation was suitably developed to simulate a neuro interventional environment, it is readily adaptable to simulate procedures related to other medical fields. We hope that our study may aid in the selection of appropriate instruments.

\section{Acknowledgements}

This research was supported by Basic Science Research Program through the National Research Foundation of Korea (NRF) funded by the Ministry of Education (2013R1A1A2011620), by the Fusion Technology Development Project funded by the Korean Small and Medium Business Administration (Project no. S2175879) and by research funds of Chonbuk National University in 2014.

\section{References}

[1] Molyneux A. International Subarachnoid Aneurysm Trial (ISAT) Collaborative Group. International subarachnoid aneurysm trial (ISAT) of neurosurgical clipping versus endovascular coiling in 2143 patients with ruptured intracranial aneurysms: a randomised trial. Lancet. 2002; 360(9342):1267-1274.

[2] Wiebers DO, Whisnant JP, Huston J, Meissner I, Brown RD, Piepgras DG, Forbes GS, Thielen K, Nichols D, O'Fallon WM, Peacock J, Jaeger L, Kassell NF, Kongable-Beckman GL, Torner JC, et al. Unruptured intracranial aneurysms: natural history, clinical outcome, and risks of surgical and endovascular treatment. Lancet. 2003; 362(9378): 103-110.

[3] Rüfenacht DA, Ohta M, Yilmaz H, Miranda C, Ruiz DS, Abdo G, Lylyk P, et al. Current concept of endovascular aneurysm treatment and about the role of stents for endovascular repair of cerebral arteries. Schweizer Archiv Für Neurolgie Und Psychiatrie. 2004; 155(7): 348-352.

[4] Tanimoto M, Arai F, Fukuda T, Itoigawa K, Hashimoto M, Takahashi I, Negoro M, et al. Telesurgery system for intravascular neurosurgery. Lecture Notes in Computer Sciences. 2000; 1935(2000): 29-39.

[5] Arai F, Fujimura R, Fukuda T, Negoro M, et al. New catheter driving method using linear stepping mechanism for intravascular neurosurgery. Proceedings of the IEEE International Conference on Robotics \& Automation. 2002; 29442949.

[6] Jayender J, Patel RV, Nikumb S, et al. Robot-assisted catheter insertion using hybrid impedance control. Proceedings of the IEEE International Conference on Robotics and Automation. 2006; 607-612.

[7] Krombach GA, Mahnken A, Tacke J, Staatz G, Haller S, Nolte-Ernsting CA, Meyer J, Haage P, Günther RW, et al. US-guided nephrostomy with the aid of a magnetic field-based navigation device in the porcine pelvicaliceal system. Journal of Vascular and Interventional Radiology. 2001; 12(5): 623-628.

[8] Chung AJ, Edwards PJ, Deligianni F, Yang GZ, et al. Freehand cocalibration of optical and electromagnetic trackers for navigated bronchoscopy. Medical Imaging and Augmented Reality. 2004; 3150: 320-328.

[9] Ellsmere J, Stoll J, Rattner D, Brooks D, Kane R, Wells W, Kikinis R, Vosburgh K, et al. A navigation system for augmenting laparoscopic ultrasound. Medical Image Computing and Computer-Assisted Intervention. 2003; 2879: 184191.

[10] Kindratenko VV. A survey of electromagnetic position tracker calibration techniques. Virtual Reality. 2000; 5(3): 169182.

[11] Nakada K, Nakamoto M, Sato Y, Konishi K, Hashizume M, Tarnura S, et al. A rapid method for magnetic tracker calibration using a magneto-optic hybrid tracker. Medical Image Computing and Computer Assisted Intervention. 2003; 2879: $285-293$.

[12] Ikits M, Brederson JD, Hansen CD, Hollerbach JM, et al. An improved calibration framework for electro-magnetic tracking devices. Proceedings of the Virtual Reality 2001 Conference. 2001: 1-8.

[13] Chuang VP, Lawrence DD, Richli WR, Lee YY, Charnsangavej C, Wallace S, et al. A large lumen microcatheter for oncologic intervention. Cardiovascular and Interventional Radiology. 1995; 18(4): 265-268.

[14] Hori S, Matsushita M, Narumi Y, Fuijta M, Tomoda K, Marukawa T, Kuroda C, Kozuka T, et al. Microcatheter with a ball tip for hepatic embolization and intra-arterial chemotherapy. European Journal of Radiology. 1989; 9(1): 44-47.

[15] Kikuchi Y, Strother CM, Boyer M, et al. New catheter for endovascular interventional procedures. Radiology. 1987; 165(3): 870-871.

[16] Komiyama M, Yasui T, Fu Y, Yagura H, Baba M, Hakuba A, Nishimura S, et al. A new microcatheter system for endovascular treatment of cerebral arteriovenous malformation. Surgical Neurology. 1989; 32(2), 91-97. 
[17] Rufenacht D and Merland JJ. A new and original microcatheter system for hyperselective catheterization and endovascular treatment without risk of arterial rupture, Journal of Neuroradiology. 1986; 13(1): 44-54.

[18] Yu CH, Ohta M, Kwon TK, et al. Study of parameters for evaluating the pushability of interventional devices using box-shaped blood vessel biomodels made of PVA-H or silicone. Bio-Medical Materials and Engineering. 2014; 24(1): 961-968.

[19] Yu CH and Kwon TK. Study of parameters for evaluating flow reduction with stents in a sidewall aneurysm phantom model. Bio-Medical Materials and Engineering. 2014; 24(6): 2417-2424.

[20] Nambu M. Elastomer including high water content. Kobunshi Kako. 1983; 32: 523-531.

[21] Millon LE, Mohammadi H, Wan WK, et al. Anisotropic polyvinyl alcohol hydrogel for cardiovascular applications. Journal of Biomedical Materials Research. Part B, Applied Biomaterials. 2006; 79(2): 305-311.

[22] Hyon SH, Cha WI, Ikada Y, et al. Preparation of poly (vinyl alcohol) hydrogels by low temperature crystallization of the aqueous poly (vinyl alcohol) solution. Kobunshi Ronbunshu. 1989; 46: 673-680.

[23] Ohkura M, Kanaya T, Kaji K, et al. Gels of poly (vinyl alcohol) from dimethyl-sulfoxide water solutions. Polymer.1992; 33(17): 3686-3690.

[24] Lyoo WS, Yeum JH, Kwon OW, Shin DS, Han SS, Kim BC, et al. Rheological properties of high molecular weight (HMW) syndiotactic poly (vinyl alcohol) (PVA)/HMW atactic PVA blend solutions. Journal of Applied Polymer Science. 2006; 102(4): 3934-3939.

[25] Yu CH, Kosukegawa H, Mamada K, Kuroki K, Takashima K, Yoshinaka K, Ohta M, et al. Development of in vitro tracking system with poly (vinyl alcohol) hydrogel for catheter motion. Journal of Biomechanical Science and Engineering. 2010; 5(1): 11-17.

[26] Schmidt W, Lanzer P, Behrens P, Topoleski LD, Schmitz KP, et al. A comparison of the mechanical performance characteristics of seven drug-eluting stent systems. Catheterization and Cardiovascular Interventions. 2009; 73(3): 350-360.

[27] Mamada K, Kosukegawa H, Fridrici V, Kapsa P, Ohta M, et al. Friction properties of PVA-H/steel ball contact under water lubrication conditions. Tribology International. 2011; 44(7): 757-763.

[28] Mamada K, Kosukegawa H, Fridrici V, Kapsa P, Ohta M, et al. Friction properties of poly (vinyl alcohol) hydrogel: Effects of degree of polymerization and saponification value. Tribology Letters. 2011; 43(2): 241-251.

[29] Zemzemi F, Bensalem W, Rech J, Dogui A, Kapsa P, et al. New tribometer designed for the characterisation of the friction properties at the tool/chip/workpiece interfaces in machining. Tribotest. 2008; 14: 11-25. 